prolapsus was constant, even when the patient was lying on her back.

Case I2. Miss M., aged 33, has been an invalid for eight years; has been under medical care most of the time. Very weak and nervous, unable to be upon her feet any length of time, and incapable of exercise of any sort. Suffers with constant pain in pelvis, which is greatly increased at the menstrual periods, particularly in uterus and left ovary. Pro. fuse leucorrhœa. Constant bearing down, and great distress in lower abdomen when on her feet. On local examination found complete anteversion with anteflexior of first degree. As all sorts of pessaries and other means of treatment had been employed without success, I determined to see what could be done for a case of this sort by Alexander's operation.

Operation, May $3 r$. Found both ligaments, which were rather small in size. After freeing ligaments from their attachments, and before making traction upon them, I introduced one finger into the vagina and placed it upon the fundus of the uterus, which lay under the pubes, while the cervix was in the hollow of the sacrum. With the finger of the right hand in this position, I made traction with my left hand upon the two ligaments simultaneously. I felt the uterus dragged upward away from the finger of the right hand, it being lifted fully two inches upward and backward, where it was retained, the ligaments being secured over a rubber plate as in preceding cases. The wounds were closed in the usual manner. Since the operation the patient has been entirely relieved of the bearing down pain which she previously suffered more or less, even when lying in bed, although, of course, the result of the operation cannot be determined until she gets upon her feet.

It seems to me that this case demonstrates not only the utility of Alexander's operation in cases of extreme anteversion, but also the erroneous character of the views which have heretofore been held by some gynecologists, in attributing anteversion to abnormal shortening of the round ligaments. Thomas gives shortening of the round ligaments as one of the causes of anteversion, and in speaking of the changes which occur in the uterine ligaments in cases of anteversion, he further states: "In anteversion the utero-sacral ligaments are generally shortened, and there is no doubt that the round ligaments are similarly altered." The fact that the uterus was lifted bodily out of its abnormal position and drawn backward to its normal position by the round ligaments, and that it was found necessary to shorten the ligaments fully three inches, shows that anteversion as the result of abnormal shortness of the round ligaments is an utter impossibility. It would also seem to demonstrate that shortening of the ligaments does not occur in anteversion-at least that a longer period than eight years is necessary even for the beginning of a change of this sort.

It would seem that a consideration of the anatomical relations of the round ligaments, the internal abdominal ring and the uterus, would be a sufficient demonstration of the ability of the ligaments to raise the womb to its normal position, and the impossibility of anteversion occurring as the result of shorten-

ing of the ligaments. The point at which the round ligaments enter the inguinal canal is halfway between the spine and pubes, and the anterior superior spine of the crest of the ilium is opposite the middle of Poupart's ligament; so that, with the uterus in anteversion, the action of the ligaments is calculated to restrain the organ from extreme anteversion. I am inclined to the belief that extreme anteversion cannot occur without elongation of the ligaments.

[Since this paper was written, the patient has left the surgical ward, and has now been upon her feet for several weeks. The uterus remains in perfect position, and she is quite relieved of the distressing "bearing down" of which she previously suffered. I feared that when the patient should get upon her feet, the uterus would rapidly return to its old position under the pubes; but, to my surprise, the fundus has been steadily retreating from a half anteverted position until, at the present date, August I, the uterus is in a position which would be perfectly normal were it not for the fact that the slight anteflexion which existed still remains. It is necessary to introduce the finger to its full length in order to be able to reach the fundus.]

\title{
THE RELATION BETWEEN ERYSIPELAS AND PUER- PERAL FEVER, CONSIDERING ERYSIPELAS BOTH AS AN ACUTE AND A LATENT DISEASE.
}

Read before the Section on Obstetrics and Gynecology, at the Thirty-eighth Annual Meting of the American Medical Association held at Chicago, Fune, 1887 . BY A. MACLAREN, M.D., OF ST. PAUL, MINN.

Before proceeding to the discussion of this subject let me present to you, in as concise a form as possible, my definition for the term puerperal fever. It is not a specific fever, as Dr. F. Barker would have us believe, but a disease due to the absorption, by a puerperal patient of some septic poison; this pois. on being absorbed either by the lymphatics or the veins at the site of some breach of continuity in the parturient canal. It is then, in short, nothing more or less than septic fever, showing the same pathological changes and giving the same clinical symptoms as are seen in different surgical patients who have suffered from septic infection.

In the year I 886 there were published two particularly valuable works on the subject of Obstetrics. The first of these, Cazeau and Tarnier's "Theory and Practice of Obstetrics," with its 1,200 pages, does not make the slightest reference to the relation existing between Erysipelas and Puerperal Fever. In Dr. Mundé's appendix, however, there is this note bearing upon the subject. "That the exanthemata cause puerperal fever I do not believe, for while after labor the material organism has lessened power to oppose the approach of disease, and is less able to withstand its inroads, scarlatina or other exanthem, when it appears at this time, still preserves its distinctive characteristics, though perhaps running an 
unusually malignant course; yet we cannot call it puerperal fever." As Dr. Mundé makes no mention of erysipelas, I understand that he here includes it with the exanthemata.

The second work to which I refer is Dr. Lusk's: "Science and Art of Midwifery." There we find a very exhaustive résumé of all the latest theories and investigations as to the nature and causation of puerperal fever, which Professor Lusk sums up in these words. "Thus we find in surgical fever, in puerperal fever, in diphtheria and in erysipelas, the presence of a common element which links them together, and which establishes the relationship which has so long been recognized as existing between these various processes."

This connecting link of which Dr. Lusk speaks is the chain-like micrococci which up to the present time have resisted all efforts at differentiation. Among the workers and authorities in this field, he quotes such men as Koch, Lukomski, Doléris, Samuel and Virchow, the last named author upholding this theory of bacterial origin in these words, "Especially in this connection are to be mentioned the diphtheritic process and the erysipelatous, especially erysipelas malignum. The granular deposit in diphtheritically affected tissues, of which I formerly spoke, has more and more proved to be of a para sitic character." I have as you will see simply reiterated Dr. Lusk's arguments and for two reasons, I. I do not feel myself competent to discuss the bacterial element of the disease, and, 2. I whish to show the wide difference of opinion between the two schools as shown by these two eminent teachers, differences which would lead them widely apart in the treatment of puerperal fever and especially in the prophylactic treatment of the disease.

Before leaving this part of my subject let me call your attention to Dr. Joseph Kucher's work on "Puerperal Convalescence." Dr. Kucher takes the position that Semmelweiss' theory for the causation of puerperal fever is the only true standard, and that the septic poison which produced the disease is probably the chain-like micrococcus. In several places Dr. Kucher takes the position that the poison of erysipelas can produce puerperal fever, mentioning cases to prove his position which $I$ shall refer to later.

There are many surgeons who contend that ery. sipelas is essentially a skin disease, with more or less severe constitional symptoms, and that we must have the skin eruption or else the disease cannot be erysipelatous in nature. In Holmes' "System of Surgery," we find this sentence: "It has been objected by $\operatorname{Sir} W$. Lawrence, who in this supports the views of Vogel and Hildenbrand, that to justify the notion of the mucous and serous membranes being affected by erysipelas, it must be shown that the same phenomena are observable in them as are seen in the skin." Holmes answers these objections in these words: "Besides diffuse cellular inflammation, there are many diseases which do not produce the characteristic cutaneous inflammation of erysipelas, yet are probably closely allied to it, as e. g.; phlebitis, both external and internal, inflamed absorbents and puerperal fever. The same may be said of some forms of inflammation of the mucous and serous membranes. For though the inflammation of the skin is absent, yet if the constitutional coliditions be the same, they must be regarded as pathologically identical with erysipelas, however the expression of the condition may differ; just as in syphilis, the same virus may produce simple chancre or phagedenic sore, or various forms of cutaneous diseases, or deep seated in. flammation." The clinical fact that a puerperal woman my have a localized erysipelas of the skin in some part of the body, and not have puerperal fever is to my mind no proof that erysipelas cannot cause puerperal fever. It is simply the woman's good fortune, for if the bacillus had beer planted in the par. turient canal instead of on the skin, the woman would have suffered from child.bed fever instead of the much less dangerous skin disease.

Allow me to offer in support of this theory a few cases in which the poison which produced the attack of puerperal fever seems to have been directly referable to an acute attack of erysipelas of the skin, and not due to the poison of another puerperal case.

Case 1 . Some years ago Dr. A. J. Stone, of St. Paul, was called to take the case of Mrs. R., who was just starting in confinement. Mrs. Dr. Stone had been ill for the preceeding 24 hours suffering with pain in the back, face and eyes, with rise of both temperature and pulse. Wednesday morring Dr. Stone delivered Mrs. R., the labor being normal, $O$. A. position, with no apparent injury to the parturient canal. Thursday Mrs. R. had a mild chill, but with no rise in temperature, accompanied however by profuse sweating. Had severe pains in back, side and head, and considerable nausea. Friday, another chill, but still no rise in temperature. Saturday severe chill followed by temperature of $105^{\circ}$, and on this day the lochia for the first time became scant and very offensive. Intra-uterine injections given regularly every every two or three hours for the next six days, and on the roth day after delivery the temper. ature became normal and patient went on to a complete recovery. At this time there was no puerperal fever in St. Paul, and neither Dr. Stone nor his nurse had seen a case of this disease for over a year. Dr. Stone gave up the rest of his practice for the time, and attended solely to these two patients, Mrs. R. and his wife. He now feels that the attack of puerperal fever was simply puerperal erysipelas carried directly from Mrs. Stone to his patient Mrs. B.

Case 2.-I was called in consultation to see a case of puerperal fever by Dr. M., of St. Paul, who prefers to withhold his name. Dr. M. was attending a case of facial erysipelas when called on March 4 th to deliver Mrs. D., of St. Paul. Labor normal, O. A. position. 48 hours after delivery Mrs. D. had a severe chill with a temperature immediately afterwards of $105^{\circ} \mathrm{F}$., pulse I20. Chill every day. Lochia suppressed and rather offensive. When I saw the patient she was suffering from a well marked case of puerperal pyæmia. Examination showed slight tear of the perineum; lochia very scant and offensive. Temperature $103.5^{\circ} \mathrm{F}$, pulse 130 . Patient died on the I6th of March, having developed metastatic abscesses in left ankle and in lungs. The nurse had 
not attended a case of puerperal fever for over a rise in temperature and developed erysipelas upon year. Dr. M. had not seen a case of puerperal the leg, starting from the lower angle of the wound. trouble for over six months, and both had attended There was no erysipelas in the hospital, nor in the any number of confinement cases in the meantime, practice of Dr. Wheaton or myself. The nurse, all of whom had recovered without a single septic however, one of the best and cieanest nurses whom 1 symptom.

Dr. Ambrose Guichard revorts a case bearing directly upon this subject in the Archices de Tocologie, July, 1885. Mrs. D. æt. 27 , delivered for the $4^{\text {th }}$ time, Jan. 17,1885 . Labur normal but rapid. Jan. scalp.

2oth, patient had a chill, fever, diarrhoea, tenderness of abdomen, suppression of lochia, and became delirious. Despite antuphogistic treatment and intrauterine douches, patient died on Jan. 3 oth of general puerperal peritonitis. 'The husband of this woman, who occupied the same bed at night, had facial erysipelas when first seen by I)r. Guichard, eruption having developed three days before the delivery of his wife. The midwife who saw Mrs. D. immediately after delivery made no examination and had not been in any way exposed to puerperal fever.

Dr. Kucher. makes the following quotation from Tyler Smith: "In another case a medical man was in constant attendance upon a patient suffering from gangrenous erysipelas, and between Jan. 8th and Mar. $22 \mathrm{~d}$ he attended the Jabors of ten women; all had puerperal fever, and eight of the patients died." This was in a town of moderate size; no other practitioners in the place were known to have had cases of puerperal fever. Several hundred similar cases are recorded.

Thus far in my review of this subject I have simply gone over the ground which has been so often argued pro and con throughout the medical world during the past few years. Now let me ask your attention to another phase of this subject. If the basterial origin of erysipelas be true, zehy is it that certain persons seem predisposed to innumerable attacks of this disease? We have all seen many sibjects in whom a slight indisposition brought on an acute attack of erysipelas and almost always located in the same area of skin surface, and these attacks without apparent exposure. Is not the answer to my question, that the first attack of erysipelas has never been cured and that the bacillus still lives in the skin ready to break out into a fresh attack upon the slightest provocation? It seems to me that this must be the answer, for, the cases of predisposition, of which every text-book speaks, and which we have all seen, are too numerous to allow of the supposition that each one of them has been repoisoned. If you grant me this. then you must agree with me in the belief that a person who has suffered from an attack of erysipelas, and in whom the disease is dormant, or never has been cured, can communicate the disease to an open wound or to a puerperal woman.

I have among my records a few surgical cases which seem to allow of such an explanation.

Case I.-Mr. W., operated upon at St. Joseph's Hospital Dec. 7, I886, by Dr. C. A. Wheaton, of St. Paul, for the removal of a large sarcomatous growth of the right thigh. Every antiseptic precaution was used both during and after the operation. Four days after the operation the patient had a chill, some few months previous; she has shown no signs of erysipelas since, but I have no doubt that she still has the microbe of erysipelas living in her face or

Case 2.-Oct. 4, I 886, Miss. B., operated upon by Dr. Millard, of Stillwater, Minn., at St. Joseph's Hospital, St. Paul, for the removal of a $40 \mathrm{lb}$. congenital perineal tumor diagnosticated to be fatus in fotu. The operation was performed in the new wing of the Hospital which had just been finished. She was placed in a room never before occupied, with new bed and bedding, no erysipelas being then present in any part of the Hospital. The patient was placed under the care of the same nurse of whom I have just spoken. This nurse had been performing the ex. ecutive duties of the hospital for several preceding months, and was only placed upon duty on account of the enlargement of the service and the scarcity of assistants. The patient did remarkably well until the evening of the sixth day, when she had a severe chill. The next succeeding day she developed erysipelas in the wound, which spread from there to the buttock and thigh of the right side. She passed into a septic condition, from which she died on the $35^{\text {th }}$ day, her physical condition corresponding very closely to that of a fatal case of puerperal septicæmia. Case 3.-Occurred at the same time on the person of a young lady upon whom Dr. Wheaton had performed an Alexander's operation, and who was under the care of this same nurse; the wound upon the left side, which was almost entirely closed, seeming to be the starting point for the eruption which spread to the labia and inside of the thigh on the left leg. There was no erysipelas in the Hospital nor in the practice of any of the physicians who were connected with either of these cases. I have called the attention of both Dr. Millard and Dr. Wheaton to this theory for the causation of these cases of erysipelas, and they have both agreed with me in the strong probability, at least, of the truth of my explanation. In looking back it seems that ever since the attack of facial erysipelas in this nurse, now over three years ago, ever since that time erysipelas has followed her, affecting apparently without cause her surgical patients. Through the kindness of Dr. Beal, of St. Paul, I am able to present a number of puerperal cases which come in the direct line of argument.

Dr. Beal suffered from a very severe attack of facial erysipelas in the winter of 1885 . In October, I 885, Dr. Beal delivered Mrs. G. of her first child. Breech presentation. I saw Mrs. G., in consultation with Dr. Beal, and applied forceps to the after coming head. The labor was normal in every respect. After-examination showed laceration of cervix on the right side. On the third day Mrs. G. had a very severe chill, followed by a rise in temperature, vomiting, pain in the back, head, and abdomen, with suppression of lochia. Regular intratuterine injections and 
a stimulating treatment were instituted but Mrs. G. 2d day after delivery Mrs. H. had a very severe died 16 days after delivery of puerperal septicæmia. chill followed by a temperature of $105 \mathrm{~F}$., pulse small I never saw Mrs. G. after the night of her confine- and rapid. Lochia became scant, but not very ofment; there was no other case of puerperal septic- fensive. Suffered from pain in abdomen and back, æmia in St. Paul, so far as I could determine at that very tender on pressure all about the uterus. $3 \mathrm{~d}$ time, and I had not seen in the meantime a single day; chill followed by temperature of IO5. $5^{\circ}$ th day; case of scarlet fever, diphtheria or erysipelas; still chill in the morning, temperature kept rising all day my next case of confinement developed puerperal septicamia on the third day after delivery.

Mrs. O., ret. 2 I, primipara. Delivered Nov. 8 , 1885. Labor tedious; head would not engage. $3^{6}$ hours after the commencement of labor she was placed thoroughly under the influence of chloroform, forceps applied and the head brought well down onto the perineum. She was then allowed to deliver herself. Female child weighing 9 lbs. Slight tear of fourchette, not more than $1 / 4$ in. in depth. One hour after delivery, placenta not having come away after a thorough trial of Credés method of expression and the patient being exsanguinated, the hand was thoroughly cleansed in bi cloride mercury solution $\mathbf{r}$ - Iooo, and introduced into the vagina. Two fingers were introduced into the uterus and the adherent placenta ;removed. On the 3 d day after delivery this patient rexeloped a trute case of puerperal pyemia. Intrauterine douches and stimulant treatment were of no avail, and she died suddenly on the $15^{\text {th }}$ day from cerebral embolism. The fact that it was found necessary to introduce the hand into the uterine cavity in this case, will probably be sufficient explanation to many of my hearers for this attack of puerperal fever; but still there seems to be a strong relationship between these two cases. The same forceps used in Dr. Beal's case were used in this case. Out of 55 cases upon my obstetric records this is the only one whom I have delivered who has had the slightest septic trouble. In several of my cases just as serious operative proceedings have been found necessary, and in several the hand has been introduced into the uterine cavity.

In the spring of $1886 \mathrm{Dr}$. Beal had a succession of puerperal fever cases. The poison seeming to follow him so closely that he had at last to refuse to take any obstetric work. As he expresses it, "Every woman that I saw during that last three weeks had a chill on the third day whether I touched her or not." During all this time Dr. Beal used the most thorough and systematic antiseptic precautions: bi-chloride of mercury, baths, changing of clothes, use of nail brush and constant rinsing of the hands; but notwithstanding all this, nearly every woman had more or less puerperal trouble, with three fatal results.

Mrs. B., delivered April 2. O. A. position. Forceps delivery. The perineum was lacerated and was restored 5 hours after delivery by Drs. Ritchie and Beal. This patient had no trouble until the gth day, when she had a severe chill, followed by a high tem perature, pain in the back, head und abdomen. Her child died in convulsions on this same day. The patient had a chill every day until the I6th, when she died of puerperal septicæmia.

Dr. Beal's next case was Mrs. H., who was in the 7 th month of pregnancy. Mrs. H. nearly aborted at

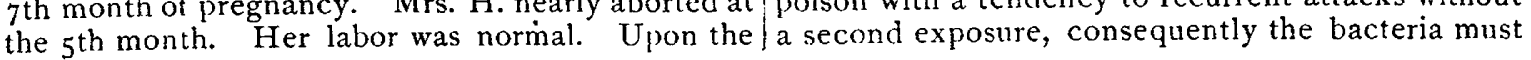


be able to live in the apparently healthy skin for an indefinite space of time.

3. That the germs of erysipelas when living in this quiescent or dormant state, although they may not be able to excite an attack of erysipelas of the skin, may still under proper conditions produce either surgical or puerperal fever.

In the first part of my paper I have presented a few cases on one side of a still unsettled subject. In the last part I have offered a theory the truth or error of which time and careful investigation alone can prove.

If my words may only cause a little greater care and the consequent saving of one material life I shall feel that I am more than repaid.

\section{A NEW METHOD OF PRODUCING LOCAL ANFS- THESIA OF THE SKIN.}

Read in the Section on Medicine, Materia Medica and Therapeutics, at the Thirty-Eighth Annual Meeting of the American Medical Association, Fune 7, 1887. BY HENRY J. REYNOLDS, M.D.,

PROFESSOR OF DERMATOLOGY, COLLEGE OF PHYSICIANS AND SURGEONS, CHICAGO; PROFESSOR OF SKIN AND GENITO-URINARY DISEASES, CHICAGo POLICLINIC; ETC, ETC.

Various means have been adopted for prodncing local anæsthesia of the skin, but heretofore none has been to any extent a success. Even cocaine, useful in most other tissues as a topical application, has absolutely no practical use as a local anæsthetic for the skin. Though local anæthesia of the deeper parts may also be produced by the method which I am about to describe, I am only prepared to speak of its merits from a dermatological standpoint at present, but am nevertheless satified that the tissues can in this way be anæsthetized to a sufficient depth to render the method applicable in many cases to general surgery.

As cocaine is the anasthetizing agent, the method therefore relates only to the manner in which the drug is applied and its absoption induced. Electricity is the means employed for this purpose. Knowing that solutions of cocaine were not readily absorbed by the skin, and therefore produce no anæsthetic effect, Dr. Wagner, of Vienna, about a year ago made experiments, the results of which he afterwards (Wien. med. Blätt., 1886, vol. 9, p. 161) presented to the Society of Physicians of Vienna in the form of a paper on that subject, with a view to as certaining a more successful method of producing anæsthesia of the skin with this drug. He found that by saturating the positive electrode of a galvanic battery with a solution of cocaine, applying it to the skin and then applying the negative electrode a short distance from the positive, with a moderate current on, that successful anæsthesia could be pro. duced. Since the publication of this article, though personally, as a rule, very skeptical regarding therapeutic innovations, $I$ have been induced to make a number of experiments with the method, and to make frequent use of it in dermatological practice with quite a degree of satisfaction; and inasmuch there- fore as I have in looking up the literature of the subject been unable to find any article tonching upon the details or results of any personal experience with the method in this country, I feel justified in writing this article, in the hope that it may elicit discussion and the personal experience of others, and be the means of bring to the more general notice of the profession of this country a procedure which I deem of much importance in nedical and surgical practice. So far as I am aware, in fact, no article on the subject has appeared in medical literature other than the one just referred to. With this apology, and without going into the details of my experiments, I trust that a brief description of the method and the results of my experience with it will be tolerated.

Battery, strength of current, etc.-I have used an eighteen cell McIntosh battery, but I think, in some cases, even a stronger current is necessary. For reasons not necessary to discuss at present the Faradic current will not answer; neither will the negative pole of the galvanic. The strength of the current must vary with the sensitiveness of the part, the size of the electrode, etc. It is always necessary to use strongest current that can be borne, which may vary all the way from four or five cells (of the McIntosh battery) to twenty-four, but for which no rule can be laid down, the only guide being the feelings of the patient and the experience and discretion of the physician. Where the skin is very dense, the part not sensitive to electricity, and the electrode large owing to the greater surface necessary to be acted upon, (which thereby renders the current more diffused, and hence in a sense not so strong) the current must be very strong, requiring perhaps 24 cells of the McIntosh instrument. The sponge on the electrode used for the solution should be of the finest and softest quality. When a strong current is used the irritation at the negative pole may be avoided by using a larger electrode.

Strength of solution.-In my experience the cocaine should not be used weaker than a five per cent. solution. It may vary, however, owing to circumstances, from a two to a twenty per cent. strength. Where the skin is thin and the part such as will tolerate a strong current, a two to five per cent. solution will answer. On the other hand, if the part be so sensitive that only a weak current can be employed, as in portions of the face, the solution to be effectual must not be less than a ten per cent. one.

Mode of Asplication. - Saturate the positive electrode with the solution and place it directly upon the part to be anæsthetized. Place the negative electrode well saturated with water on some point near by. A more remote point, as the hand of the opposite side, for instance, will answer, but it will take longer time and a stronger solution to get the effect than when placed near by. In working abour the face I think it is better to hold the negative electrode in the hand than to apply it to the face near the positive, as in this way a stronger current will be better tolerated.

It is well for the operator to familiarize himself with the method by experiments upon his own person. The electrodes should be kept firmly pressed 